\title{
Antidamping Torques from Simultaneous Resonances in Ferromagnet-Topological Insulator-Ferromagnet Heterostructures
}

\author{
A. A. Baker, ${ }^{1,2}$ A. I. Figueroa, ${ }^{1}$ T. Hesjedal, ${ }^{2}$ and G. van der $\operatorname{Laan}^{1, *}$ \\ ${ }^{1}$ Magnetic Spectroscopy Group, Diamond Light Source, Didcot, OX11 ODE, United Kingdom \\ ${ }^{2}$ Clarendon Laboratory, Department of Physics, University of Oxford, \\ Parks Road, Oxford, OX1 3PU, United Kingdom
}

(Dated: August 24, 2018)

\begin{abstract}
We studied the magnetodynamics of ferromagnetic films coupling across a topological insulator (TI) $\mathrm{Bi}_{2} \mathrm{Se}_{3}$ layer using ferromagnetic resonance (FMR). TIs have attracted much attention across the physics community as they hold the potential for dissipationless carrier transport, extremely high spin-orbit torques, and are host to novel quantum effects. To investigate the coupling between the ferromagnetic (FM) layers, vector network analyzer (VNA)-FMR measurements of the resonance linewidth were performed as a function of bias field angle. By bringing the resonances of the two FM layers into close proximity, it was possible to observe antidamping torques that lead to a narrowing of linewidth, a characteristic of spin pumping. The element- and hence layer-specific technique of $\mathrm{x}$-ray detected ferromagnetic resonance (XFMR) was used to circumvent the difficulty of obtaining accurate fits to the two overlapping resonances in close proximity. Our results confirm that the interaction across the TI is a dynamic exchange mediated by spin pumping, as opposed to a selfcoupling of the surface state or similar, more unconventional mechanisms.
\end{abstract}

\section{INTRODUCTION}

Spin transfer in magnetic multilayers offers the possibility of a new generation of ultrafast, low-power spintronic devices. New ways to control the resonance frequency and damping in ultrathin films are actively sought, fueling the study of the precessional dynamics and interaction mechanisms in such samples. One effect that has come under particular scrutiny in recent years is the spin-transfer torque (STT), wherein a flow of spins entering a ferromagnet exerts a torque on the magnetization, inducing precession ${ }^{1}$. A flow of spin angular momentum is usually generated through a spin-polarized electrical current, but a promising alternative is the pure spin current emitted by a ferromagnet undergoing ferromagnetic resonance $(\mathrm{FMR})$. This allows spins to be transferred without a net charge flow. The physics of the generation, transmission and absorption of pure spin currents is a developing field, and holds great promise for both industrial applications and as a means to study fundamental physical phenomena in exotic materials.

The exciting physics of topological insulators (TIs) has been under intense study since their theoretical prediction $^{2}$ and experimental verification ${ }^{3-5}$. Recently, they were shown to display the quantum anomalous Hall effect after doping with magnetic impurities ${ }^{6}$, and are proposed to host image magnetic monopoles and the giant magneto-optical effect ${ }^{7-10}$. In the prototypical 3D TI $\mathrm{Bi}_{2} \mathrm{Se}_{3}$ a large spin-orbit interaction leads to a band inversion in the bulk and the formation of topologically protected surface states (TSS), with fully spin-polarized counter-propagating conduction channels that are robust against scattering from non-magnetic impurities ${ }^{11}$. Spinmomentum locking suggests the possibility of very long spin-flip scattering lifetimes and the ability to generate ultra-high spin-orbit torques ${ }^{12-14}$. It has been predicted that the TSS can exert a torque on spins in a neighboring ferromagnet (FM) through exchange coupling ${ }^{15}$. However, in order to realize these prospects the magnetodynamics of the TSS must be studied and such TI-FM heterostructures fabricated. While angle-resolved photoemission spectroscopy (ARPES) has been extremely successful at identifying $\mathrm{TIs}^{4,5}$, transport measurements are limited due to large bulk conductivities that make unambiguously identifying the surface state contributions challenging $^{16,17}$. It is therefore beneficial to apply a wider range of techniques to the study of TIs, aiming to focus more closely on the spin degrees of freedom present in the TSS.

FMR offers an attractive means to investigate the coupling of ferromagnets to TIs, using the pure spin current emitted at the resonance condition to probe the bulk and surface state. Recently, studies of such effects have begun to emerge through electrical transport and inverse spin-Hall effect measurements ${ }^{14,18,19}$, demonstrating the great potential of TIs for incorporation into spintronic devices such as spin valves.

This paper describes FMR experiments designed to assess the spintronic potential of TIs, using $\mathrm{Co}_{50} \mathrm{Fe}_{50}(30 \mathrm{~nm}) / \mathrm{Bi}_{2} \mathrm{Se}_{3}\left(t_{\mathrm{TI}}\right) / \mathrm{Ni}_{81} \mathrm{Fe}_{19}(30 \mathrm{~nm})$ heterostructures with TI interlayer thickness $t_{\mathrm{TI}}=4,6,8$.

Using vector network analyzer (VNA)-FMR measurements we found in a previous study ${ }^{20}$ on these heterostructures a linear increase of the damping parameter, $\alpha$, for both the NiFe and CoFe layers as a function of $t_{\mathrm{TI}}$ : In the $t_{\mathrm{TI}}$ range from 0 to $20 \mathrm{~nm}, \alpha$ for NiFe gradually increases from $7.2 \times 10^{-3}$ to $9.8 \times 10^{-3}$ and $\alpha$ for CoFe gradually increases from $7.4 \times 10^{-3}$ to $12 \times 10^{-3}$. While these measurements showed that the TI layer can absorb a spin current, the nature of the coupling between the two ferromagnetic layers remained unclear. Phase-resolved measurements using x-ray detected ferromagnetic resonance (XFMR), which are able to distinguish between static 
and dynamic exchange coupling ${ }^{21}$, did provide in this case no conclusive answer ${ }^{20}$. While a dynamic interaction through spin pumping is a likely candidate, another interaction mediated by the topological surface state is also a possibility. Therefore, another experimental approach is required to provide more information as to the origin of the interaction. In heterostructures with strong magnetocrystalline anisotropy it is possible to use the angular dependence of the resonance condition to tune the separation of the two resonances, and identify the spin pumping $^{22}$.

To study the coupling between the FM layers in more detail, measurements of resonance linewidth were performed as a function of bias field angle. By bringing the resonances of the two layers into close proximity, it was possible to observe antidamping torques that lead to a narrowing of linewidth. However, this is complicated by the difficulty of obtaining accurate fits to the overlapping two resonances in close proximity. To overcome this difficulty we performed element- and hence layer- specific measurements using XFMR.

\section{DAMPING AND SPIN PUMPING}

The study of magnetic relaxation processes in thin films and nanoscale devices has become increasingly important in recent years, spurred by the interest in phenomena such as spin-transfer torques and vortex core dynamics $^{23,24}$. Relaxation of the excited magnetic state can proceed by a number of different mechanisms, including coupling to the crystal lattice ${ }^{25}$, dissipation into the magnetic subsystem through two-magnon scattering ${ }^{26,27}$, and spin pumping ${ }^{1,28}$. The resonance linewidth functions as a sensitive probe of damping, and many studies have examined the interplay of layer structure, crystal quality and magnetization alignment in determining the precessional damping ${ }^{29-32}$.

\section{A. Gilbert damping}

The resonance linewidth, $\Delta H$, contains both intrinsic (Gilbert) and extrinsic (non-Gilbert) contributions, and is given by ${ }^{33}$

$$
\Delta H=\frac{4 \pi f}{\gamma} \alpha+\Delta H_{0},
$$

with $\alpha$ the (dimensionless) Gilbert damping parameter and $\Delta H_{0}$ the extrinsic broadening. Gilbert damping in pure metals is primarily caused by spin-orbit interaction, scattering the excitations from phonons or magnons ${ }^{34}$. Ultimately, this mechanism allows transfer of energy from the spin subsystem of the sample to the lattice. There are several mechanisms by which it may be enhanced, for example, through the introduction of rareearth impurities ${ }^{35-37}$, the presence of an adjacent rareearth layer ${ }^{38}$, or through spin pumping, wherein angular momentum is pumped out of an on-resonance ferromagnetic layer ${ }^{39}$.

\section{B. Spin currents}

The generation and detection of spin currents is at the foundation of spintronics, being integral to many proposals for new memory and logic devices ${ }^{24,35,40-42}$. The pure spin current emitted by a ferromagnet undergoing $\mathrm{FMR}^{43}$ is one candidate for generating such currents, and offers the opportunity to study these effects in the absence of a charge current. A spin current can persist across a normal metal (NM), and in a trilayer structure either return to the first $\mathrm{FM} / \mathrm{NM}$ interface, or else flow through to a second NM/FM interface. If it is not reflected here, it crosses the interface and is absorbed by the FM, inducing precession through $\mathrm{STT}^{44}$. Such spin pumping can be observed by measuring increased damping (through increased FMR linewidth) due to outflow of angular momentum from the source $\mathrm{FM}^{28,39}$, by inverse spin Hall effect in the sink layer ${ }^{45}$, or layer-specific measurements of precession or spin accumulation using x-ray magnetic circular dichroism (XMCD) ${ }^{20,21,46-48}$.

In the case of a FM1/NM/FM2 structure, the second FM layer acts as a spin sink for the spin current driven out of the first FM layer. The absorbed spin current exerts a torque on the static magnetization in FM2, which can lead to precession of magnetization even when the resonance condition is not met. The addition of a second scattering interface and a high-efficiency spin sink modifies the spin pumping equations significantly. In this case the Landau-Lifshitz-Gilbert (LLG) equation becomes ${ }^{46}$

$$
\begin{aligned}
-\frac{\partial \mathbf{m}^{i}}{\partial t}= & \mathbf{m}^{i} \times\left[\gamma^{i} \mathbf{H}_{\mathrm{eff}}^{i}-\left(\alpha_{0}^{i}+\alpha_{i i}^{i}\right) \frac{\partial \mathbf{m}^{i}}{\partial t}\right] \\
& +\alpha_{i j}^{i} \mathbf{m}^{j} \times \frac{\partial \mathbf{m}^{j}}{\partial t},
\end{aligned}
$$

where the superscript denotes the magnetic layer number, $\alpha_{0}^{i}$ the intrinsic Gilbert damping parameter, $\alpha_{i i}^{i}$ damping due to angular momentum pumped out of layer $i$, and $\alpha_{i j}^{i}$ (anti)damping due to angular momentum driven into layer $i$ from layer $j$. The antidamping torque is only significant when both layers are simultaneously on-resonance, otherwise $\partial \mathbf{M}^{j} / \partial t \approx 0$ and its effects are negligible. Damping is highest when the spin current can cross the spacer layer and be efficiently absorbed by the second FM layer. As the thickness of the spacer layer increases the spin pumping decreases due to increasing backflow. The increase in damping reaches its minimum value when the spacer layer is thicker than the spin coherence length, and no current reaches FM2 (Ref. 49). 


\section{EXPERIMENTAL}

\section{A. Sample fabrication}

Samples were prepared using molecular-beam epitaxy (MBE). The evaporation sources were calibrated using a quartz crystal microbalance and beam-flux monitor. First, the $\mathrm{MgO}$ substrate was annealed at $700^{\circ} \mathrm{C}$ to clean the surface and reduce the surface roughness. Next, $30 \mathrm{~nm}$ of $\mathrm{Co}_{50} \mathrm{Fe}_{50}$ was co-evaporated from $\mathrm{Fe}$ and $\mathrm{Co}$ electron-beam evaporators. The substrate temperature was held at $300^{\circ} \mathrm{C}$ during this stage. Strong streaks and Kikuchi lines were observed by reflection high-energy electron diffraction (RHEED), indicative of high crystalline quality. Samples were then transferred into a chalcogenide MBE chamber for growth of the $\mathrm{Bi}_{2} \mathrm{Se}_{3}$ layers. Growth of the TI layers was performed according to growth protocols previously developed for thin film topological insulators, see, e.g., Refs. 50 and 51. Bi and Se were then evaporated from Knudsen cells for stoichiometric growth of $\mathrm{Bi}_{2} \mathrm{Se}_{3}$ at a substrate temperature of $200^{\circ} \mathrm{C}$. RHEED patterns reveal the formation of a crystalline mosaic domain pattern. The $\mathrm{Bi}_{2} \mathrm{Se}_{3}$ thickness was varied between $4-8 \mathrm{~nm}$. The samples were then transferred back to the metal MBE chamber, and a top FM layer of $\mathrm{Ni}_{81} \mathrm{Fe}_{19}(30 \mathrm{~nm})$ deposited at room temperature $(\sim 300 \mathrm{~K})$, to avoid damaging the $\mathrm{Bi}_{2} \mathrm{Se}_{3}$ layer. This leads to the formation of polycrystalline $\mathrm{Ni}_{81} \mathrm{Fe}_{19}$, which is desirable as it ensures fully isotropic magnetic behavior. Samples were grown with an interlayer thickness of $t_{\mathrm{TI}}=4,6,8$. Finally, the samples were capped with $5 \mathrm{~nm}$ $\mathrm{Cu}$, to prevent oxidation.

\section{B. VNA-FMR}

FMR measurements were done using a Rhode \& Schwartz ZVB20 vector network analyzer (VNA) in the frequency range $0.5-20 \mathrm{GHz}$. Samples were mounted face-down on a coplanar waveguide (CPW) of characteristic impedance $50 \Omega$ and placed in a vector electromagnet, capable of applying a static field of up to $0.5 \mathrm{~T}$ in any direction. Real and imaginary components of the microwave transmission parameter, $S_{12}$, were measured as a function of field vector (strength and angle) and frequency. The resulting resonances were fitted using asymmetric Lorentzians to extract the resonance frequency and line width. All measurements were performed at room temperature.

Example field-frequency transmission maps along the easy and hard axes are shown in Fig. 1 for a sample with $t_{\mathrm{TI}}=8 \mathrm{~nm}$. Strong resonances are observed from both layers, with the CoFe displaying a strong four-fold cubic anisotropy $\left(K c_{\|}=41.1 \pm 0.2 \mathrm{~kJ} \mathrm{~m}^{-3}\right)$, and the $\mathrm{NiFe}$ having a much weaker two-fold uniaxial anisotropy. The magnetocrystalline anisotropy parameters and $g$ factors for the $\mathrm{CO}_{50} \mathrm{Fe}_{50}$ and $\mathrm{Ni}_{81} \mathrm{Fe}_{19}$ modes of the heterostructures, determined by fitting the angle- and field-
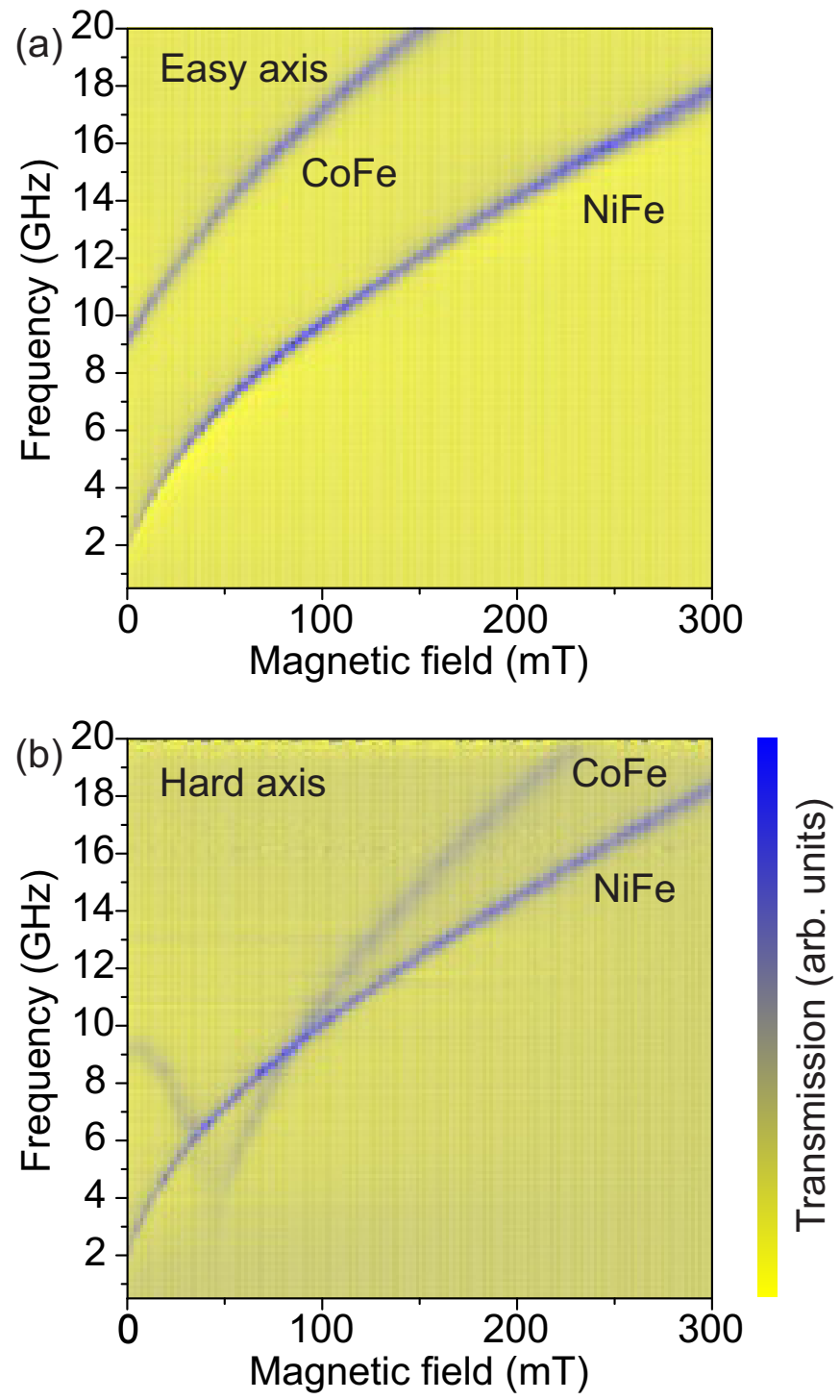

FIG. 1. Field vs frequency transmission maps for bias field applied along the easy (a) and hard (b) axes of the CoFe layer of a sample with $t_{\mathrm{TI}}=8 \mathrm{~nm}$. Two strong resonance modes are observed, which can be attributed to the CoFe and NiFe layers. The anisotropic mode comes from the crystalline $\mathrm{Co}_{50} \mathrm{Fe}_{50}$.

dependent FMR, can be found in Ref. 52 .

\section{XFMR}

XFMR offers element- and thus layer-specific measurement of the precessional dynamics of each FM layer on a picosecond timescale ${ }^{21,52-55}$. In this technique, the magnetization of the sample in a magnetic field is pumped by a radio-frequency ( $\mathrm{RF}$ ) field to generate a precessing moment (i.e., FMR), which is probed using the XMCD effect $^{56,57}$. The XMCD signal is proportional to the pro- 
jection of the x-ray beam direction onto the magnetization.

The sample is mounted on a CPW and driven by the applied RF field while under a DC bias field. A schematics of our XFMR setup can be found in Ref. 52. The incident x-rays impinge the sample through a hole in the signal line of the CPW. The x-ray beam has an incidence angle of $35^{\circ}$ with respect to the plane of the sample, to ensure that the XMCD is sensitive to the larger in-plane component of the magnetization precession. The x-ray absorption was measured in transmission geometry by detecting the x-ray excited optical luminescence emerging from the $\mathrm{MgO}$ substrate, using a photodiode mounted behind the sample ${ }^{52}$.

The XFMR experiments were performed on beamline I10 at the Diamond Light Source, UK, using the portable octupole magnet system (POMS) from the Magnetic Spectroscopy Group. The setup allows to measure excitation frequencies up to $12 \mathrm{GHz}$. The XMCD signals were detected at the Co $L_{3}(\sim 778 \mathrm{eV})$ and Ni $L_{3}(\sim 853$ $\mathrm{eV}$ ) absorption edges.

\section{ANTIDAMPING TORQUES FROM SIMULTANEOUS RESONANCE}

The additional damping caused by spin pumping arises due to the energy loss when spins are pumped out of the on-resonance layer. In the same way, this outflow of energy can induce precession in the off-resonance layer, through the spin transfer torque, when it is absorbed. These effects enter the LLG equation [Eq. (2)] as the damping and antidamping constants, $\alpha_{i i}^{\mathrm{sp}}$ and $\alpha_{i j}^{\mathrm{sp}}$, respectively. If both layers are simultaneously onresonance, then the antidamping from the incoming spin current can partially compensate the additional damping from the outgoing spin current. Experimentally, this is observed as a decrease in resonance linewidth as the separation of the resonances of the two layers approach one another. Heterostructures with a strong magnetocrystalline anisotropy allow us to use the angular dependence of the resonance condition to change the separation between the two resonances, and identify signs of spin pumping ${ }^{22}$. In practice this method is somewhat more complicated due to the difficulty of obtaining accurate fits to the two overlapping Lorentzian resonances in close proximity. This is illustrated in Fig. 2, showing the change in resonance field of the two modes as a function of excitation frequency. Note that spin pumping is not the only source of angular variation of resonant linewidth; mosaic broadening and two-magnon scattering can also play a role.

\section{A. VNA-FMR results}

The steps to evaluate the presence of antidamping torques are shown in Fig. 3 for a sample with $t_{\mathrm{TI}}$
$=8 \mathrm{~nm}$, demonstrating the difficulty of obtaining unambiguous data. In particular, significant additional linewidth broadening occurs when the magnetization is canted away from the static bias field, as happens at low fields in the vicinity of a magnetocrystalline hard axis. An example of this can be seen in Fig. 3(e) below $8 \mathrm{GHz}$. This effect can cause the apparent resonant linewidth to increase by a factor of ten or more, but it does not have a physical relation to the spin pumping process. For this reason, such data regions must be excluded.

The correlation between mode separation and linewidth at $10 \mathrm{GHz}$ is shown in Fig. 3(f). The linewidth of the $\mathrm{CoFe}$ resonance decreases with decreasing separation, which suggests the presence of a spin-pumping mediated dynamic interaction. The linewidth decreases when the spin torques exerted by the pure spin current partially cancel. However, a slight increase in damping is observed in the $\mathrm{Ni}_{81} \mathrm{Fe}_{19}$, which in theory should show the same trend as in the $\mathrm{Co}_{50} \mathrm{Fe}_{50}$. The discrepancy could be related to an intrinsic damping anisotropy, exacerbated by dissimilar spin pumping efficiencies from the two layers, as evidenced by a lower spin mixing conductance observed for the $\mathrm{Co}_{50} \mathrm{Fe}_{50} / \mathrm{TI}$ interface, indicating that spins are less readily pumped from the $\mathrm{Co}_{50} \mathrm{Fe}_{50}$.

The effects of mode proximity on the linewidth at $8.9 \mathrm{GHz}$ is shown in Fig. 4 for $t_{\mathrm{TI}}=4,6$, and $8 \mathrm{~nm}$. In all cases the linewidth of the CoFe decreases as the modes move towards one another, dropping to a minimum $\sim 3.5 \mathrm{mT}$, suggesting that this is the intrinsic linewidth for the bare film when the spin pumping damping cancels. However, the linewidth of the NiFe is largely unchanged across the same range, and even shows a slight increase as the modes approach crossover, which contradicts the previous interpretation of antidamping torques. The change in linewidth of the CoFe could then instead come from misfitting caused by overlap, or else artificial broadening due to the canting of magnetization away from the bias field. The data indicates the presence of antidamping torques, in turn suggesting that the interaction across the TI is mediated by spin pumping, but cannot be conclusive due to the aforementioned difficulty interpreting the data.

\section{B. Layer-resolved measurements}

XFMR offers a solution to some of the problems inherent in measuring overlapping resonances, as its elementselectivity ensures that only one layer is measured at a time. Further, it is less sensitive to magnetization canted away from the bias field, due to reduced projection along the x-ray beam in turn reducing the dynamic XMCD signal. Results of separation-dependent linewidth at $8 \mathrm{GHz}$ for samples with $t_{\mathrm{TI}}=8 \mathrm{~nm}$ are plotted in Fig. 5 . The data show a narrowing of resonant linewidth with decreasing mode separation for $t_{\mathrm{TI}}=8 \mathrm{~nm}$, though as with the VNA-FMR measurements, only in the CoFe layer. This suggests that the linewidth narrowing previously 

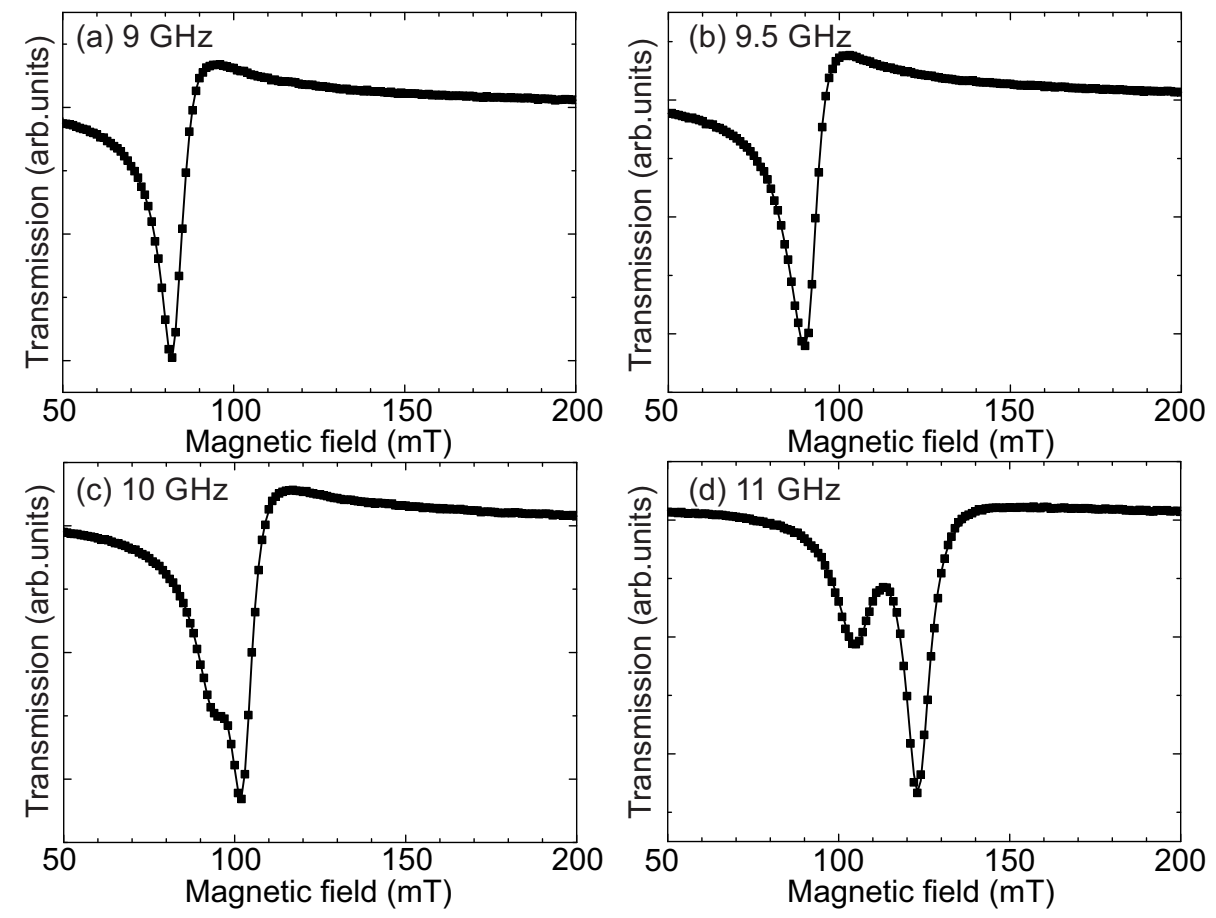

FIG. 2. VNA-FMR data for a sample with $t_{\mathrm{TI}}=8 \mathrm{~nm}$ near the hard axes, showing a change in resonance field as a function of excitation frequency. The modes completely overlap at $9 \mathrm{GHz}$ (a), making it hard to separate individual contributions to the linewidth. The modes move apart as frequency increases (b,c), and are fully resolved at $11 \mathrm{GHz}(\mathrm{d})$. Black squares are data points, solid lines are fits to the data using two asymmetric Lorentzian curves.

observed is a genuine effect, likely stemming from simultaneous spin pumping.

However, it remains unclear why little to no variation is observed in the NiFe layer. It is possible that the problem relates to dissimilar efficiencies of spin pumping across the FM/TI interfaces. This discrepancy is also present in measurements of phase and amplitude of precession across resonance, where variations in phase take place in only one layer ${ }^{20}$. These results highlight the critical importance of high quality interfaces for spin pumping experiments, and show that there is room for further study of spin transfer in FM-TI heterostructures.

\section{CONCLUSIONS}

Measurements of resonance linewidths were carried out as a function of bias field angle to study the coupling in $\mathrm{FM} 1 / \mathrm{TI}\left(t_{\mathrm{TI}}\right) / \mathrm{FM} 2$ pseudo-spin valves. By bringing the resonances of the two FM layers into close proximity, it was possible to observe antidamping torques that lead to a narrowing of linewidth, a characteristic of spin pumping. This confirms that the interaction across the TI is a dynamic exchange coupling mediated by spin pumping, as opposed to a self-coupling of the surface state or another more unusual mechanism. Temperature dependent measurements of Gilbert damping showed no significant variation down to $10 \mathrm{~K}$ (Ref. 58), demonstrating that a suppression of the bulk conduction channels of the TI does not fundamentally alter its spin-transfer properties.

These results show the great potential of TIs for device applications, as these effects can be observed at room temperature and low magnetic fields, as well as being a fertile ground for investigation of fundamental physical phenomena. Further work is required to improve understanding of the FM/TI interface and separate bulk and surface conduction, but these initial results show that FMR, and in particular the element-specific technique of $\mathrm{XFMR}$, is a valuable tool to develop greater understanding of this new materials class.

\section{ACKNOWLEDGMENTS}

This publication arises from research funded by the John Fell Oxford University Press Research Fund. We thank Diamond Light Source for beamtime on I10 under proposal numbers SI-9210 and SI-11500, and the Research Complex at Harwell for their hospitality. A.A.B. acknowledges funding from Diamond Light Source through a joint studentship and EPSRC through a doctoral training award. 
(a)

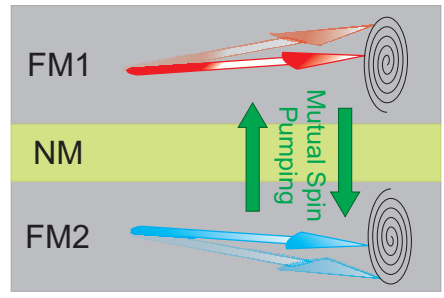

(d)

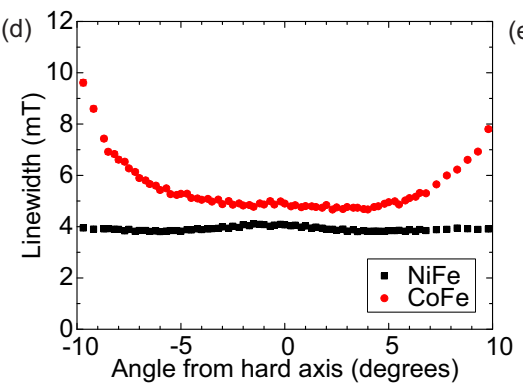

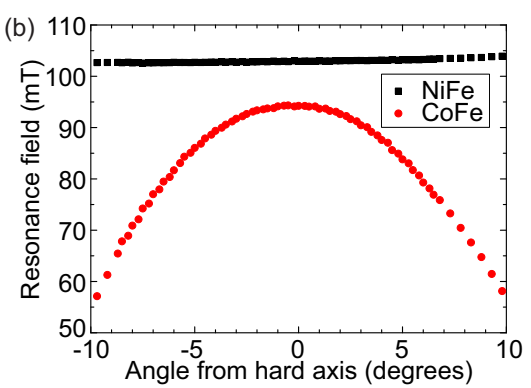
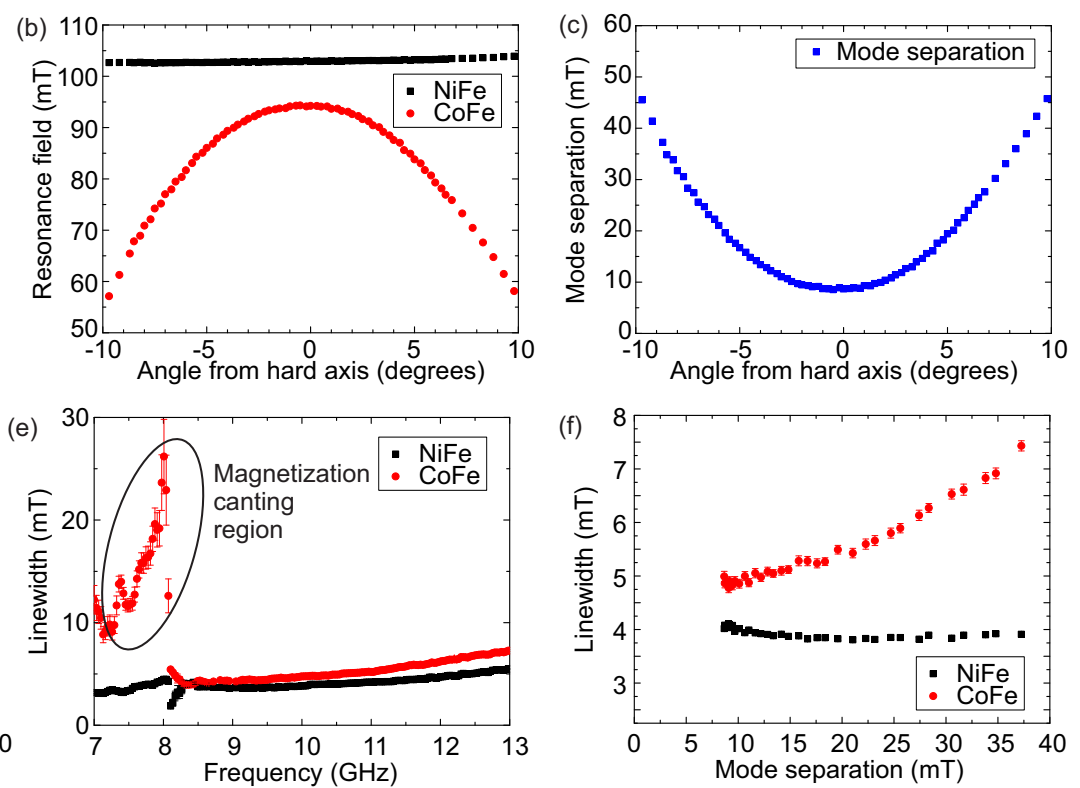

FIG. 3. Analyzing antidamping torques in FMR. The concept of mutual spin pumping is outlined in (a). Panel (b) shows the angular variation of linewidth at $10 \mathrm{GHz}$ around the magnetocrystalline hard axis of the CoFe layer, with the mode separation plotted in (c). Variation of linewidth with respect to bias field angle is shown in (d), which indicates the difficulty of separating variation due to mutual spin pumping and other relaxation mechanisms. Panel (e) shows frequency dependent linewidth performed $5^{\circ}$ away from the hard axis. Two regions of unusable data are shown. First, where overlapping resonances lead to spurious linewidths, in the region 8.0-8.5 GHz. Secondly, the canting of magnetization at low bias fields leads to the drastic increase in CoFe resonant linewidth in CoFe below $8 \mathrm{GHz}$. Taking into account these limitations, the variation of linewidth as a function of mode separation at $10 \mathrm{GHz}$ is plotted in (f). All error bars arise from uncertainty on fits to the resonances.

* Corresponding author: gerrit.vanderlaan@diamond.ac.uk

1 Y. Tserkovnyak, A. Brataas, G. E. W. Bauer, and B. I. Halperin, Rev. Mod. Phys. 77, 1375 (2005).

2 C. L. Kane and E. J. Mele, Phys. Rev. Lett. 95, 146802 (2005).

3 B. A. Bernevig, T. L. Hughes, and S.-C. Zhang, Science 314, 1757 (2006).

4 Y. L. Chen, J. G. Analytis, J.-H. Chu, Z. K. Liu, S.-K. Mo, X. L. Qi, H. J. Zhang, D. H. Lu, X. Dai, Z. Fang, S. C. Zhang, I. R. Fisher, Z. Hussain, and Z.-X. Shen, Science 325, 178 (2009).

${ }^{5}$ Y. L. Chen, J.-H. Chu, J. G. Analytis, Z. K. Liu, K. Igarashi, H.-H. Kuo, X. L. Qi, S. K. Mo, R. G. Moore, D. H. Lu, M. Hashimoto, T. Sasagawa, S. C. Zhang, I. R. Fisher, Z. Hussain, and Z. X. Shen, Science 329, 659 (2010).

${ }^{6}$ C.-Z. Chang, J. Zhang, X. Feng, J. Shen, Z. Zhang, M. Guo, K. Li, Y. Ou, P. Wei, L.-L. Wang, Z.-Q. Ji, Y. Feng, S. Ji, X. Chen, J. Jia, X. Dai, Z. Fang, S.-C. Zhang, K. He, Y. Wang, L. Lu, X.-C. Ma, and Q.-K. Xue, Science 340, 167 (2013).

7 X.-L. Qi, R. Li, J. Zang, and S.-C. Zhang, Science 323, 1184 (2009).

8 W.-K. Tse and A. H. MacDonald, Phys. Rev. Lett. 105, 057401 (2010).

9 J. R. Williams, A. J. Bestwick, P. Gallagher, S. S. Hong, Y. Cui, A. S. Bleich, J. G. Analytis, I. R. Fisher, and D. Goldhaber-Gordon, Phys. Rev. Lett. 109, 056803 (2012).

10 D. Kong, Y. Chen, J. J. Cha, Q. Zhang, J. G. Analytis, K. Lai, Z. Liu, S. S. Hong, K. J. Koski, S.-K. Mo, Z. Hussain, I. R. Fisher, Z.-X. Shen, and Y. Cui, Nature Nanotech. 6, 705 (2011).

11 J. E. Moore, Nature 464, 194 (2010).

12 M. H. Fischer, A. Vaezi, A. Manchon, and E.-A. Kim, arXiv preprint arXiv:1305.1328 (2013).

13 Y. Fan, P. Upadhyaya, X. Kou, M. Lang, S. Takei, Z. Wang, J. Tang, L. He, L.-T. Chang, M. Montazeri, G. Yu, W. Jiang, T. Nie, R. N. Schwartz, Y. Tserkovnyak, and K. L. Wang, Nat. Mater. 13, 699 (2014).

14 Y. Shiomi, K. Nomura, Y. Kajiwara, K. Eto, M. Novak, K. Segawa, Y. Ando, and E. Saitoh, Phys. Rev. Lett. 113, 196601 (2014).

15 T. Yokoyama, J. Zang, and N. Nagaosa, Phys. Rev. B 81, 241410 (2010).

16 Z. Ren, A. A. Taskin, S. Sasaki, K. Segawa, and Y. Ando, Phys. Rev. B 82, 241306 (2010).

17 C. H. Li, O. M. J. van't Erve, J. T. Robinson, Y. Liu, L. Li, and B. T. Jonker, Nat. Nanotechnol. 9, 218 (2014).

18 J. Tian, I. Childres, H. Cao, T. Shen, I. Miotkowski, and Y. P. Chen, Solid State Commun. 191, 1 (2014).

19 A. R. Mellnik, J. S. Lee, A. Richardella, J. Grab, P. J. Mintun, M. H. Fischer, A. Vaezi, A. Manchon, E.-A. Kim, N. Samarth, and D. C. Ralph, Nature 511, 449 (2014). 

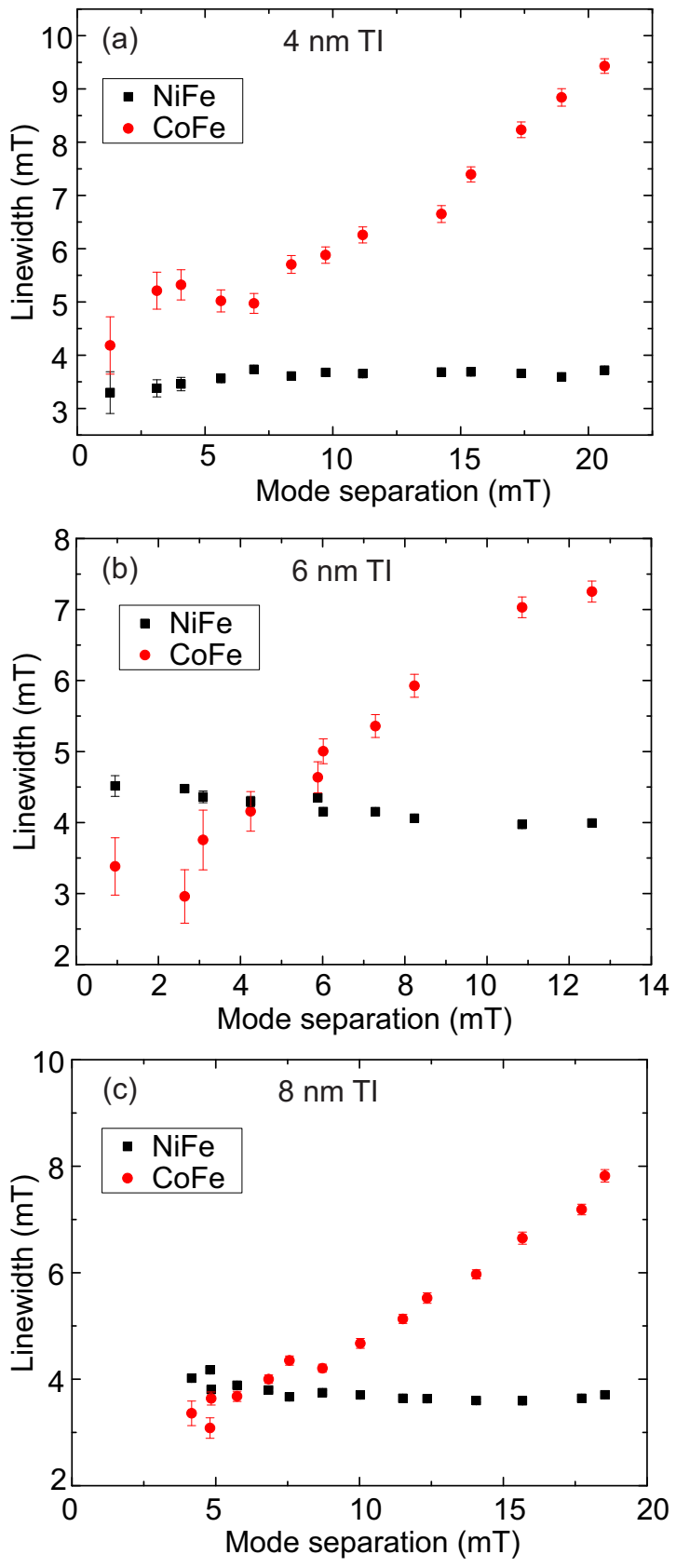

FIG. 4. Variation of linewidth at $8.9 \mathrm{GHz}$ as a function of separation of the modes for $t_{\mathrm{TI}}=4,6,8 \mathrm{~nm}$, as shown in (a), (b), and (c), respectively] A systematic variation is only evident in the CoFe layer.

20 A. A. Baker, A. I. Figueroa, L. J. Collins-McIntyre, G. van der Laan, and T. Hesjedal, Sci. Rep. 5, 7907 (2015).

${ }^{21}$ G. van der Laan, J. Electron Spectrosc. Relat. Phenom. 220, 137 (2017).

22 B. Heinrich, Y. Tserkovnyak, G. Woltersdorf, A. Brataas, R. Urban, and G. E. Bauer, Phys. Rev. Lett. 90, 187601 (2003).

23 T. A. Ostler, R. Cuadrado, R. W. Chantrell, A. W. Rushforth, and S. A. Cavill, Phys. Rev. Lett. 115, 067202

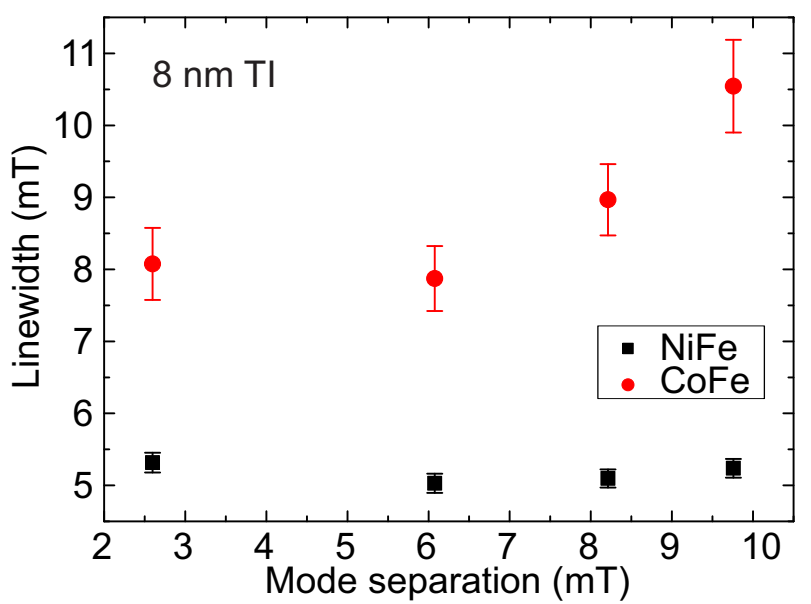

FIG. 5. Linewidth of resonance measured by XFMR as a function of mode separation for both layers in structures with $t_{\mathrm{TI}}=8 \mathrm{~nm}$. A decrease in linewidth is observed for the CoFe layer. Error bars on points arise from uncertainty on fits to the real and imaginary components of resonance.

(2015).

24 J. C. Sankey, Y.-T. Cui, J. Z. Sun, J. C. Slonczewski, R. A. Buhrman, and D. C. Ralph, Nat. Phys. 4, 67 (2008).

25 G. Woltersdorf, Spin-pumping and two-magnon scattering in magnetic multilayers, Ph.D. thesis, Simon Fraser University (2004).

${ }^{26}$ G. Woltersdorf, M. Buess, B. Heinrich, and C. H. Back, Phys. Rev. Lett. 95, 037401 (2005).

27 K. Zakeri, J. Lindner, I. Barsukov, R. Meckenstock, M. Farle, U. von Hörsten, H. Wende, W. Keune, J. Rocker, S. S. Kalarickal, K. Lenz, W. Kuch, K. Baberschke, and Z. Frait, Phys. Rev. B 76, 104416 (2007).

28 S. Mizukami, Y. Ando, and T. Miyazaki, Phys. Rev. B 66, 104413 (2002).

29 Y. Tserkovnyak, A. Brataas, and G. E. W. Bauer, Phys. Rev. Lett. 88, 117601 (2002).

30 M. Tokaç, S. A. Bunyaev, G. N. Kakazei, D. S. Schmool, D. Atkinson, and A. T. Hindmarch, Phys. Rev. Lett. 115, 056601 (2015).

31 T. Chiba, G. E. W. Bauer, and S. Takahashi, Phys. Rev. B 92, 054407 (2015).

${ }^{32}$ K. Lenz, H. Wende, W. Kuch, K. Baberschke, K. Nagy, and A. Jánossy, Phys, Rev. B 73, 144424 (2006).

33 S. S. Kalarickal, P. Krivosik, M. Wu, C. E. Patton, M. L. Schneider, P. Kabos, T. Silva, and J. P. Nibarger, J. Appl. Phys. 99, 093909 (2006).

34 B. Heinrich, R. Urban, and G. Woltersdorf, J. Appl. Phys. 91, 7523 (2002).

35 Z. Diao, Z. Li, S. Wang, Y. Ding, A. Panchula, E. Chen, L.-C. Wang, and Y. Huai, J. Phys.: Condens. Matter 19, 165209 (2007).

36 A. A. Baker, A. I. Figueroa, G. van der Laan, and T. Hesjedal, AIP Adv. 5, 077117 (2015).

37 G. Woltersdorf, M. Kiessling, G. Meyer, J.-U. Thiele, and C. H. Back, Phys. Rev. Lett. 102, 257602 (2009).

38 M. K. Marcham, W. Yu, P. S. Keatley, L. R. Shelford, P. Shafer, S. A. Cavill, H. Qing, A. Neudert, J. R. Chil- 
dress, J. A. Katine, E. Arenholz, N. D. Telling, G. van der Laan, and R. J. Hicken, Appl. Phys. Lett. 102, 062418 (2013).

39 Y. Tserkovnyak, A. Brataas, and G. E. W. Bauer, Phys. Rev. B 66, 224403 (2002).

40 N. Locatelli, V. Cros, and J. Grollier, Nat. Mater. 13, 11 (2014).

41 S. Urazhdin, V. E. Demidov, H. Ulrichs, T. Kendziorczyk, T. Kuhn, J. Leuthold, G. Wilde, and S. Demokritov, Nat. Nanotechnol. 9, 509 (2014).

42 J. A. Katine and E. E. Fullerton, J. Magn. Magn. Mater. 320, 1217 (2008).

43 A. Brataas, Y. Tserkovnyak, G. E. W. Bauer, and B. I. Halperin, Phys. Rev. B 66, 060404 (2002).

44 M. D. Stiles and A. Zangwill, Phys. Rev. B 66, 014407 (2002).

45 O. Mosendz, J. E. Pearson, F. Y. Fradin, S. D. Bader, and A. Hoffmann, Appl. Phys. Lett. 96, 022502 (2010).

46 M. K. Marcham, L. R. Shelford, S. A. Cavill, P. S. Keatley, W. Yu, P. Shafer, A. Neudert, J. R. Childress, J. A. Katine, E. Arenholz, N. D. Telling, G. van der Laan, and R. J. Hicken, Phys. Rev. B 87, 180403 (2013).

47 R. Kukreja, S. Bonetti, Z. Chen, D. Backes, Y. Acremann, J. A. Katine, A. D. Kent, H. A. Dürr, H. Ohldag, and J. Stöhr, Phys. Rev. Lett. 115, 096601 (2015).

48 J. Li, L. R. Shelford, P. Shafer, A. Tan, J. X. Deng, P. S. Keatley, C. Hwang, E. Arenholz, G. van der Laan, R. J. Hicken, and Z. Q. Qiu, Phys. Rev. Lett. 117, 076602 (2016).
49 B. Kardasz and B. Heinrich, Phys. Rev. B 81, 094409 (2010).

50 L. J. Collins-McIntyre, S. E. Harrison, P. Schönherr, N.-J. Steinke, C. J. Kinane, T. R. Charlton, D. Alba-Veneroa, A. Pushp, A. J. Kellock, S. S. P. Parkin, J. S. Harris, S. Langridge, G. van der Laan, and T. Hesjedal, Europhys. Lett. 107, 57009 (2014).

${ }^{51}$ L. J. Collins-McIntyre, W. Wang, B. Zhou, S. C. Speller, Y. L. Chen, and T. Hesjedal, Phys. Status Solidi B 252, 1334 (2015).

52 A. I. Figueroa, A. A. Baker, L. J. Collins-McIntyre, T. Hesjedal, and G. van der Laan, J. Magn. Magn. Mater. 400, 178 (2015).

${ }^{53}$ G. B. G. Stenning, L. R. Shelford, S. A. Cavill, F. Hoffmann, M. Haertinger, T. Hesjedal, G. Woltersdorf, G. J. Bowden, S. A. Gregory, C. H. Back, P. A. J. de Groot, and G. van der Laan, New J. Phys. 17, 013019 (2015).

54 A. A. Baker, A. I. Figueroa, C. J. Love, S. A. Cavill, T. Hesjedal, and G. van der Laan, Phys. Rev. Lett. 116, 047201 (2016).

55 A. A. Baker, A. I. Figueroa, D. Pingstone, V. K. Lazarov, G. van der Laan, and T. Hesjedal, Sci. Rep. 6, 35582 (2016).

56 G. van der Laan, J. Phys.: Conf. Ser. 430, 012127 (2013).

57 G. van der Laan and A. I. Figueroa, Coord. Chem. Rev. 277, 95 (2014).

58 A. A. Baker, A. I. Figueroa, G. van der Laan, and T. Hesjedal, Results Phys. 6, 293 (2016). 